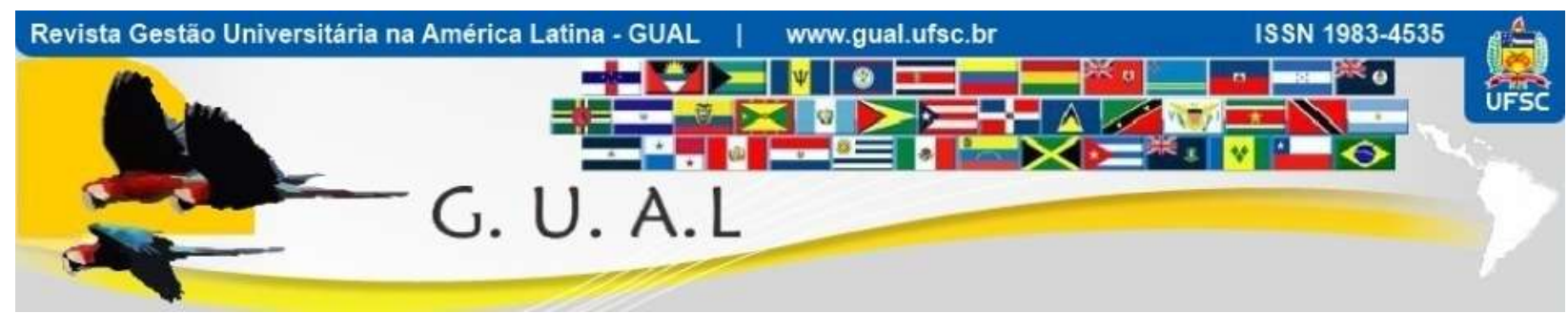

DOI: http://dx.doi.org/10.5007/1983-4535.2020v13n2p140

\title{
GOOGLE ACADÊMICO: UMA OPÇÃO PARA ANÁLISE DE CITAÇÕES DOS PERIÓDICOS BRASILEIROS DE CONTABILIDADE?
}

\section{GOOGLE SCHOLAR: AN OPTION FOR CITATION ANALYSIS OF BRAZILIAN ACCOUNTING JOURNALS?}

\author{
Sandro Vieira Soares, Doutor \\ http://orcid.org/0000-0001-7076-4936 \\ sandrovs@usp.br \\ Universidade do Sul de Santa Catarina | Programa de Pós-Graduação em Administração \\ Florianópolis | Santa Catarina | Brasil \\ Raimundo Nonato Lima Filho, Doutor \\ https://orcid.org/0000-0003-4953-5260 \\ rnlfilho@gmail.com \\ Autarquia Educacional do Vale do São Francisco | Universidade de Pernambuco | Campus Salgueiro \\ Petrolina | Pernambuco | Brasil \\ Silvia Pereira de Castro Casa Nova, Doutora \\ http://orcid.org/0000-0003-1897-4359 \\ silvianova@usp.br \\ Universidade de São Paulo | Faculdade de Economia, Administração e Contabilidade \\ São Paulo | São Paulo | Brasil
}

Recebido em 07/junho/2019

Aprovado em 06/fevereiro/2020

Publicado em 01/maio/2020

Sistema de Avaliação: Double Blind Review 


\title{
RESUMO
}

Este artigo tem por objetivo identificar se o Google Acadêmico é uma opção para análise de citações dos artigos publicados nos periódicos brasileiros de contabilidade. Para isto, foram coletadas as citações de 1.810 artigos publicados entre 2007 e 2012 por quinze revistas brasileiras de contabilidade; a coleta foi feita via Google Scholar nos meses de abril anos de 2014, 2015 e 2016. O percentual de artigos não encontrados pelo Google Acadêmico apresentou uma tendência de queda ao longo do período: de 2,8\% em abril de 2014 para $0,9 \%$ em abril de 2016. A frequência de artigos cuja contagem de citações caiu entre 2014 e 2016 foi de 1,6\% da amostra. O coeficiente de correlação de postos de Spearman entre o Fator de Impacto de 5 anos do Spell e a média de citações dos artigos publicados entre 2008 e 2012 por revista é de $r_{s}=0,918$, embora o Google Acadêmico tenha recuperado um número de citações de 3 a 9 vezes maior por revista do que o Spell. A contribuição deste artigo permite que os pesquisadores e instituições conheçam as limitações e possibilidades do uso do Google Acadêmico nas pesquisas de análise de citações de artigos brasileiros e na análise do impacto das pesquisas.

Palavras-chave: Citações. Revista. Contabilidade. Fator de Impacto.

\begin{abstract}
This paper aims to identify whether Google Scholar is an option in analyzing the citations of papers published in Brazilian accounting journals. To that end, we collected the citations of 1,810 papers published between 2007 and 2012 by fifteen accounting journals; the collection was done via Google Scholar, accessed in the months of April of the years 2014, 2015, and 2016. The percentage of papers not found by Google Scholar showed a downward trend over the period: from $2.8 \%$ in April 2014 to $0.9 \%$ in April 2016. The frequency of papers whose citation counts fell between 2014 and 2016 was 1.6\% of the sample. The Spearman rank correlation coefficient between Spell's 5-year Impact Factor and the average citation count of the papers published between 2008 and 2012 for each journal is rs $=0.918$; however, for Google Scholar, the retrieved citation counts per journal were between 3 to 9 times higher than Spell. The contributions of this article allow researchers and institutions to know the limitations and possibilities of the use of Google Scholar for citation analysis of Brazilian articles and for the analysis of the research impact.
\end{abstract}

Keywords: Citations. Journal. Accounting. Impact Factor. 


\section{INTRODUÇÃO}

O número de vezes que um artigo é citado constitui uma informação importante em diferentes dimensões. Na dimensão da comunicação científica o número de vezes que um artigo é citado constitui um indicador do quanto aquele artigo está ressoando na comunidade científica. Um artigo com muitas citações é interpretado tendo alto impacto. Na dimensão pessoal do pesquisador, o número de vezes que seus artigos são citados é compreendido como medida de eficiência e qualidade da sua carreira científica. Um pesquisador que contabiliza muitas citações, ou pelo menos mais citações que os seus pares, é tido como autoridade em sua linha de pesquisa e aumenta o seu prestígio e influência. Na dimensão da avaliação de periódicos, uma revista ter os artigos publicados bem citados a torna um canal de comunicação importante para uma determinada comunidade acadêmica, aumentando seu prestígio e credibilidade em tarefa de divulgação científica.

Todas as interpretações acima não estão à salvo de críticas e têm sido altamente questionadas. O que é mais difícil de ser discutido é o fato de que a academia tem despendido muita energia e atenção para na análise de citações durante o século XX e início do século XXI. Desde que Eugene Garfield criou o Fator de Impacto, em 1960, tornando-se um dos pais da bibliometria, muitas pesquisas têm sido feitas acerca da análise de citações. A maior parte delas se debruça a analisar os milhões de artigos e citações mapeados pelas grandes bases como o Web of Science, da Thomson Reuters ou a Scopus, da Elsevier.

Todavia, a quase totalidade dos mais de 40 periódicos brasileiros de Contabilidade não está indexada nestas duas bases de dados, o que inviabiliza as tradicionais abordagens de análise de citação no âmbito da academia em contabilidade no Brasil. No entanto, a não indexação dos periódicos nas grandes bases de dados não é característica específica da comunidade brasileira de pesquisadores de Contabilidade. Isto ocorre em outras áreas e em outros países e estas comunidades tem testado a possibilidade do uso do Google Acadêmico como uma alternativa para medir citações de artigos, impacto de revistas e ressonância de pesquisadores.

Diante deste contexto, este artigo apresenta a seguinte questão de pesquisa: O Google Acadêmico é uma opção para a análise de citações dos periódicos brasileiros de contabilidade? O objetivo desta pesquisa é, portanto, analisar o quão confiável é o uso dos dados de citações oriundos do Google Acadêmico em pesquisas de análise de citações dos periódicos brasileiros de contabilidade. 
Para tanto, foi desenvolvida uma pesquisa longitudinal iniciada em abril de 2014 e concluída em julho de 2017, acerca do comportamento das citações de 1,8 mil artigos publicados entre os anos de 2007 e 2012 pelos 15 periódicos científicos mantidos por programas de pós-graduação stricto sensu em Contabilidade ou Controladoria existentes em 2013 no Brasil.

Este artigo está dividido em cinco seções: na Introdução apresenta-se a contextualização e a questão de pesquisa; na Revisão de literatura são elencados os achados de pesquisas similares que tangenciam o objetivo da presente pesquisa; nos Procedimentos metodológicos é apresentada as amostras investigadas e os processos de coleta de dados; na Análise dos resultados são discutidos os achados da pesquisa; e, por fim, as Conclusões da pesquisa são sintetizadas na quinta seção.

\section{REVISÃO DE LITERATURA}

Uma série de pesquisas estimam a confiabilidade da contagem de citações feitas por meio do Google Acadêmico. Estas pesquisas normalmente estimam a confiabilidade da contagem de citações do Google Acadêmico e as correlacionam as contagens das citações recuperadas pelo ISI Web of Science (WoS) ou Scopus.

Jacso (2005a) realizou uma comparação entre WoS, Scopus e Google Acadêmico sendo um dos primeiros trabalhos do tipo. A coleta de dados ocorreu em abril e maio de 2005 com artigos de biblioteconomia e ciência da informação. O Google Acadêmico recuperou, aproximadamente, um terço das citações do WoS e do Scopus. O autor concluiu criticando que o Google Acadêmico não utilizava operadores de pesquisa booleana.

Jacso (2005b) realizou também uma comparação entre WoS e Google Acadêmico. A coleta de dados foi realizada em dezembro de 2004 para artigos da revista Asian Pacific Journal of Allergy and Immunology. Os resultados apontaram que, embora o Google Acadêmico tenha encontrado 680 artigos e o WoS encontrado 675, o Google Acadêmico identificou 595 citações e o WoS identificou 1.355, ou seja, pouco menos da metade.

Noruzi (2005) também desenvolveu um comparativo entre o WoS e o Google Acadêmico. O autor coletou dados em setembro de 2005, usando operadores booleanos. O autor aponta que o Google recuperou 50\% mais citações que o WoS, mas ressalvou que o Google Acadêmico não realizava pesquisas em idiomas que usam letras chinesas, persas, árabes ou japonesas. 
Pauly e Stergiou (2005) compararam o WoS e o Google Acadêmico. A comparação foi feita entre 114 artigos de 11 áreas do conhecimento publicados entre 1925 e 2004 . Os autores concluíram que o WoS recuperou mais citações que o Google Acadêmico para os artigos publicados entre 1925 e 1999, mas não houve diferença significativa nas citações dos artigos publicados entre 2000 e 2004. Os autores apontam os potenciais benefícios oriundos da gratuidade do Google Acadêmico frente ao monopólio do ISI Web of Science.

Bakkalbasi et al. (2006) realizaram uma comparação entre WoS, Scopus e Google Acadêmico. A coleta de dados foi realizada entre 7 e 12 de novembro de 2005 para artigos das áreas de oncologia e física da matéria condensada publicados em 1993 e 2003. O WoS recuperou mais citações na área de física da matéria condensada e para artigos de oncologia publicados em 1993. O Scopus recuperou mais citações para artigos de oncologia de 2003. O Google Acadêmico foi o que recuperou menos citações, entre as três opções.

Kousha e Thelwall (2007) realizaram uma comparação entre o Google Web/URL, o Google Acadêmico e o WoS. A coleta de dados ocorreu em setembro e outubro de 2005. Os autores detectaram que existe uma ampla correlação entre as citações do Google Acadêmico e do WoS. Para os autores, o Google Acadêmico é uma ferramenta possivelmente mais adequada para análise de citações em ciências sociais que nas chamadas "ciências duras".

Meho e Yang (2007) fizeram uma comparação entre WoS, Scopus e Google Acadêmico. A coleta de dados ocorreu em março de 2006, com 30 artigos da Current Science. Os autores afirmam que o Google Acadêmico se destaca na cobertura de anais de eventos e jornais de idiomas diferentes do inglês, aumentando significativamente as taxas de citação, mas criticaram severamente a baixa usabilidade do buscador.

Schroeder (2007) realizou uma comparação entre WoS e Google Acadêmico em termos de recursos e capacidade de cobertura. O autor concluiu que o WoS apresenta como vantagens o fato de cobrir mais revistas de prestígio e possuir ferramentas de busca avançada enquanto o Google Acadêmico cobre uma área maior de anais de eventos, livros e outros formatos de reportes de pesquisa, em idioma que não o inglês, e recupera mais citações.

Bar-Ilan (2008) comparou WoS, Scopus e Google Acadêmico. A coleta de dados foi realizada em novembro de 2006 para o índice $\mathrm{H}$ de 40 pesquisadores israelenses. A autora identificou que a diferença da capacidade de recuperação de citações para cálculo do índice H entre o Google Acadêmico e as demais bases varia conforme a área. $\mathrm{O}$ índice $\mathrm{H}$ do Google 
Acadêmico para áreas de matemática e computação é 30\% maior que nas demais bases, enquanto que na área de física de alta energia é 30\% menor.

Kousha e Thelwall (2008) compararam o WoS e Google Acadêmico. A coleta de dados ocorreu em janeiro de 2006, por duas pessoas. Os autores identificaram que o Google Acadêmico cobre uma variedade de documentos mais ampla e, por isso, recupera taxas de citações superiores para artigos publicados em revistas de acesso aberto. Afirmam, ainda, que essa amplitude da cobertura Open Access pode impulsionar o movimento de acesso aberto.

Bornmann et al. (2009) fizeram uma comparação entre Google Acadêmico, WoS, Scopus e Chemical Abstracts. Os autores apontam que as contagens de citação do WoS, Scopus e Chemical Abstracts são muito similares entre si e diferentes das taxas do Google Acadêmico. Corroboram as críticas de Jacso (2005 apud PERKEL, 2005) de que "o Google Scholar faz um trabalho realmente horrivel de conexão entre artigos citantes e artigos citados".

Kulkarni et al. (2009) fizeram uma comparação entre WoS, Scopus e Google Acadêmico. A coleta de dados foi realizada em 2 de junho de 2008 com 328 artigos de medicina, por quatro pessoas. O Scopus e Google Acadêmico recuperaram taxas maiores de citações que o WoS. A precisão na busca de citações pelo Google Scholar foi levemente menor que as demais. Os autores concluíram que o Google Acadêmico cobre livros, dissertações e teses, e revistas de acesso aberto.

Martell (2009) realizou uma comparação entre Yahoo, Google, Google Acadêmico e WoS. A coleta de dados ocorreu entre o verão e o outono de 2007 de 217 artigos publicados entre 2000 e 2006 na revista College \& Research Libraries. O autor aponta que o Yahoo e Google não possuem motores de busca adequados para recuperar citações. Por outro lado, o Google Acadêmico recuperou uma taxa média de citações para os artigos maior que o dobro da taxa recuperada pelo WoS.

Onyancha (2009) estabeleceu uma complexa avaliação dos periódicos da África Subsaariana. O autor criticou que os métodos tradicionais de análise de citações do WoS não podem ser utilizados para esses periódicos porque a maioria dos periódicos em questão não estão indexados no WoS, e a maioria das instituições africanas não pode pagar as altas taxas de assinatura do ISI. O autor concluiu que o Google Acadêmico permitiu a comparação do impacto de revistas que o WoS não cobre, permitindo uma análise com o software Publish or 
Perish que seria inviável de outra maneira. Sobre as restrições do Google Acadêmico, o autor as classifica como "retificáveis".

Bar-Ilan (2010) realizou uma comparação entre WoS, Scopus e Google Acadêmico. A coleta de dados foi feita em 22 de março de 2008 usando um único título: o livro Introduction to Infometrics. O WoS e o Scopus cobriram aproximadamente $90 \%$ das citações recuperadas um pelo outro. O Google Acadêmico cobriu 30\% menos que as citações encontradas pelos demais, porém encontrou 108 citações que não haviam sido encontradas pelos demais, o que representa um acréscimo de $30 \%$ de citações não catalogadas pelos demais. A autora alerta ainda para a baixa usabilidade do buscador.

García-Pérez (2010) comparou o WoS, PsycInfo e Google Acadêmico. A coleta de dados ocorreu em outubro e novembro de 2009, de artigos de quatro psicólogos espanhóis. Em ordem decrescente de recuperação de volume de citações estão: Google Acadêmico, PsycInfo e WoS. O autor identificou citações incorretas na ordem de $0,3 \%$ no WoS, 1,1\% no PsycInfo e 16,5\% no Google Acadêmico.

Mingers e Lipitakis (2010) realizaram uma comparação entre WoS e Google Acadêmico na área de negócios e gestão. A coleta de dados englobou artigos de três faculdades de negócios inglesas. Os autores identificaram que WoS não recupera nem metade das revistas, artigos e citações recuperados pelo Google Acadêmico. Todavia, os autores alertam para a confiabilidade e a falta de transparência do Google Acadêmico.

Sanni e Zainab (2010) desenvolveram uma avaliação das citações do Medical Journal of Malaysia. Os autores identificaram que o Google Acadêmico se mostrou uma alternativa viável para análise de citações mais ampla e menos restritiva que o WoS.

Sember, Utrobicić e Petrak (2010) fizeram uma comparação entre WoS, Scopus e Google Acadêmico. A coleta de dados foi feita entre os meses de janeiro e março de 2009 dos artigos do Croatian Medical Journal. Em ordem crescente de recuperação de citações: WoS, Scopus e Google Acadêmico, sendo que este último recuperou $22 \%$ de citações únicas, não recuperadas pelos demais sistemas.

Amara e Landry (2012) compararam o WoS e Google Acadêmico na área de negócios e gestão. A coleta de dados sobre 1.286 pesquisadores canadenses da área de negócios ocorreu entre abril e junho de 2010. Os autores concluíram que a abrangência do Google Acadêmico é muito superior à do WoS mas que, apesar disso, havia uma forte 
correspondência entre os autores mais citados no Google Acadêmico com os autores citados no WoS.

Bergman (2012) comparou o WoS, Scopus e Google Acadêmico. Os resultados mostraram que o WoS recuperou a menor taxa de citação, seguido de perto pelo Scopus. O Google Acadêmico recuperou uma taxa de citação substancialmente superior aos anteriores, mas os autores afirmam que o Google Acadêmico pode não ser tão confiável quanto os demais.

Harzing (2013) realizou uma comparação entre WoS e Google Acadêmico. A autora analisou a estabilidade, abrangência e imparcialidade do Google Acadêmico por meio de um estudo longitudinal das citações de 20 laureados com o prêmio Nobel em química, economia, medicina e física. A coleta de dados ocorreu em abril e setembro de 2011 e janeiro de 2012. A autora apontou que o Google Acadêmico mostrou estabilidade e abrangência. A taxa de erro na recuperação de artigos foi de $0,5 \%$.

Delgado-López-Cózar e Cabezas-Clavijo (2013) compararam o Google Scholar Metrics, o JCR e o Scimago Journal Rank. Os dados foram coletados em janeiro de 2013. Os autores concluem que, mesmo com baixa transparência acerca da cobertura do Google Acadêmico, ele ordena as pesquisas de forma muito similar aos demais buscadores e que, provavelmente, o recurso será o maior concorrente das versões pagas.

Chan, C. Chang e Y. Chang (2013) analisaram o desempenho de 23 periódicos de finanças, usando o Google Acadêmico. A coleta de dados aconteceu entre julho e setembro de 2011. Os autores identificaram que revistas de alto fator de impacto tendem a apresentar mediana de citações alta e taxas de artigos não citados baixa, grande proporção de artigos de líderes acadêmicos e que, em geral, membros do corpo editorial têm alto impacto nas citações. Os autores ainda identificaram que o topo do ranking de revistas, construído com base do Google Acadêmico, é similar ao dos rankings desenvolvidos em outras pesquisas.

Ortega e Aguillo (2014) fizeram uma comparação entre o Microsoft Academic Search e Google Scholar Citations. A coleta de dados ocorreu em junho de 2012. O Google Scholar Citations abrange mais documentos e citações que o Microsoft Academic Search, mas possui um viés mais forte na área de informática e ciências da computação, enquanto o Microsoft Academic Search apresentou mais equilíbrio entre áreas. Por outro lado, o Microsoft Academic Search apresenta problemas de duplicação de perfis e menor taxa de atualização. 


\section{GOOGLE ACADÊMICO: UMA OPÇÃO PARA ANÁLISE DE CITAÇÕES DOS PERIÓDICOS \\ BRASILEIROS DE CONTABILIDADE? \\ DOI: http://dx.doi.org/10.5007/1983-4535.2020v13n2p140}

A literatura acima converge para a seguinte síntese: o Google Acadêmico tem capacidade de recuperação de citações mais abrangente que seus concorrentes, até mesmo o WoS e Scopus. No entanto, ele é menos preciso que seus concorrentes. Todavia, há um grande problema para o teste da capacidade de recuperação de citações das revistas brasileiras de Contabilidade: o fato de que a maioria delas não está indexada no WoS ou no Scopus para que essa comparação possa ser feita. Apesar disso, algumas pesquisas brasileiras estão utilizando o Google Scholar como fonte de dados. É o caso de Souza, Ensslin e Ensslin (2012), Aragão, Oliveira e Lima (2014), Soares e Casa Nova (2016) e Soares (2017).

\section{PROCEDIMENTO METODOLÓGICOS}

Para desenvolver esta pesquisa foi necessário escolher uma amostra representativa de artigos publicados em revistas brasileiras de Contabilidade. Esta pesquisa foi iniciada em 2014. À época, os autores desta pesquisa julgaram que a amostra que possuiria maior consistência para participar seria composta pela lista de periódicos relacionados ou mantidos por programas de pós-graduação stricto sensu em Contabilidade e Controladoria. A lista das revistas relacionadas a programas de pós-graduação na área é apresentada na Figura 1, com a instituição de vínculo e o número de artigos publicados no período.

Figura 1 Revistas da amostra, por instituição e número de artigos na amostra

\begin{tabular}{|l|c|c|}
\hline \multicolumn{1}{|c|}{ Revista } & Instituição & N. de artigos \\
\hline BASE & Unisinos & 144 \\
\hline Brazilian Business Review - BBR & Fucape & 117 \\
\hline Contabilidade Vista e Revista & UFMG & 142 \\
\hline Contabilidade, Gestão e Governança & UnB & 115 \\
\hline Contextus - Revista Contemporânea de Economia e Gestão & UFC & 80 \\
\hline Revista Brasileira de Gestão de Negócios - RBGN & FECAP & 140 \\
\hline Revista Contabilidade \& Finanças & USP & 121 \\
\hline Revista Contemporânea de Contabilidade & UFSC & 96 \\
\hline Revista de Contabilidade da UFBA & UFBA & 91 \\
\hline Revista de Contabilidade do Mestrado em Ciências Contábeis da UERJ & UERJ & 113 \\
\hline Revista de Contabilidade e Controladoria & UFPR & 75 \\
\hline Revista de Contabilidade e Organizações & USP/RP & 129 \\
\hline Revista de Informação Contábil Uril & UFPE & 130 \\
\hline Revista Universo Contábil & FURB & 201 \\
\hline Sociedade, Contabilidade e Gestão & UFRJ & 116 \\
\hline \multicolumn{1}{|c|}{ Total } & $\mathbf{1 5}$ & $\mathbf{1 . 8 1 0}$ \\
\hline
\end{tabular}

Fonte: elaborada pelos autores (2017).

Cada uma das edições publicadas entre os anos de 2007 e 2012 pelas revistas listadas na Figura 1 foi acessada. Uma lista de todos os artigos publicados por elas foi criada em uma 
planilha eletrônica de Microsoft Excel®. A lista inicial possuía 1.812 artigos, mas foi verificado que dois artigos apareciam duas vezes na lista: o mais antigo foi publicado em 2007 e 2008 na mesma revista e o mais recente foi publicado em duas revistas diferentes em 2008. Nesse caso optou-se por eliminar a duplicidade, mantendo apenas a versão mais antiga.

O número de citações que cada artigo possuía em abril de 2014, identificado pelo Google Acadêmico, foi registrado na planilha. O mesmo procedimento foi realizado novamente em abril de 2015 e abril de 2016 para identificar o comportamento do número de citações de cada artigo ao longo do tempo. Os resultados identificados são explorados na próxima seção “Análise de Resultados”. Assim, chegou-se ao total de 1.810 artigos, relacionados por revistas pertencentes a 15 diferentes instituições que mantêm programas de pós-graduação na área.

\section{ANÁLISE DOS RESULTADOS}

A análise da capacidade do Google Acadêmico de identificar o número citações foi baseada em algumas características. A primeira delas foi do número de artigos não encontrados por essa base ao longo dos anos. Todos os artigos de nove das 15 revistas da amostra foram encontrados pelo Google Acadêmico nos três anos em que houve coleta de dados. As ocorrências de artigos não encontrados pelo buscador se restringiram às seis revistas apresentadas na Tabela 1.

Tabela 1 Número de artigos não recuperados, por ano

\begin{tabular}{l|c|c|c|c|c|c|c}
\multicolumn{1}{c|}{ Revista } & $\mathbf{A b r} / \mathbf{1 4}$ & $\mathbf{\%}$ & $\mathbf{A b r} / \mathbf{1 5}$ & $\mathbf{\%}$ & $\mathbf{A b r} / \mathbf{1 6}$ & $\mathbf{\%}$ & Total \\
\hline BASE & 0 & $0,0 \%$ & 1 & $0,7 \%$ & 0 & $0,0 \%$ & 144 \\
\hline Brazilian Business Review - BBR & 7 & $6,0 \%$ & 5 & $4,3 \%$ & 2 & $1,7 \%$ & 117 \\
\hline Revista Brasileira de Gestão de Negócios & 7 & $5,0 \%$ & 6 & $4,3 \%$ & 0 & $0,0 \%$ & 140 \\
\hline Revista de Contabilidade e Controladoria & 1 & $1,3 \%$ & 0 & $0,0 \%$ & 0 & $0,0 \%$ & 75 \\
\hline Revista de Contabilidade e Organizações & 1 & $0,8 \%$ & 0 & $0,0 \%$ & 0 & $0,0 \%$ & 129 \\
\hline Revista de Informação Contábil & 34 & $\mathbf{2 6 , 2 \%}$ & 42 & $32,3 \%$ & 15 & $11,5 \%$ & 130 \\
\hline Totais & $\mathbf{5 0}$ & $\mathbf{2 , 8 \%}$ & $\mathbf{5 4}$ & $\mathbf{3 , 0 \%}$ & $\mathbf{1 7}$ & $\mathbf{0 , 9 \%}$ & $\mathbf{7 3 5}$ \\
\hline
\end{tabular}

Fonte: elaborada pelos autores (2017).

A revista que teve mais casos de artigos não encontrados pelo Google Acadêmico foi a Revista de Informação Contábil - RIC, da UFPE. A taxa de artigos não recuperados foi de $11,5 \%$ na busca de 2016 até 32,3\% na busca de 2015. Esta revista teve problemas com o acesso ao site durante um período de vários meses o que pode ter acarretado na dificuldade do Google Acadêmico de recuperar esses artigos. A BBR e a RBGN foram as duas revistas com 
maior número artigos não recuperados na sequência, com sete artigos não encontrados cada, em 2014. Este número caiu para zero para a RBGN e para dois para a BBR em 2016. Um artigo da RCC (UFPR) e um da RCO (USP/RP) não foram recuperados em 2014, mas ambos foram encontrados nos anos seguinte assim como um artigo da revista Base que não foi encontrado em 2015.

De modo geral, as taxas de não-recuperação dos artigos foram maiores em 2014 e 2015 e menores em 2016. De aproximadamente 3\% da amostra a taxa caiu para aproximadamente 1\% em 2016. Coloca-se aqui uma sugestão para pesquisas futuras a identificação se esta taxa manteve tendência de queda em 2017 e períodos seguintes.

A segunda forma de identificar a capacidade de contagem de citações do Google Acadêmico é identificar o comportamento anômalo do número de citações ao longo do tempo. Teoricamente, o número de citações de um artigo é cumulativo e tende a crescer ou se manter constante.

A redução do número de citações de um artigo, nesta coleta de dados, só poderia ocorrer em duas situações: a primeira é, se por algum motivo, o artigo citante deixasse de existir. Por exemplo: se um artigo X, publicado no momento t, fosse citado por um artigo Y em $\mathrm{t}+1$, ao final de $\mathrm{t}+1$ o artigo contabilizaria 1 citação. No entanto, se o artigo $\mathrm{Y}$ fosse retratado, republicado com uma versão que omitisse a citação do artigo $\mathrm{X}$, ou mesmo 'despublicado', ao final de t+2 o número de citações poderia diminuir. Esta é a forma natural que o número de citações poderia cair. O mesmo ocorreria se o artigo fosse citado em qualquer documento online como uma ementa de disciplina, ou um comentário em um blog que fosse apagado.

A segunda forma seria que o algoritmo de busca de artigos do Google Acadêmico não é estável e apresenta uma oscilação de queda do número de citações excessivamente grande. Considerando que nenhuma das 15 revistas publicou retratações, erratas ou a exclusão de artigos de suas coleções durante o período da pesquisa, pode-se assumir que as reduções da contagem de citações derivam de imprecisões do Google Acadêmico. A Tabela 2 lista as quedas ocorridas por revista, entre os anos de 2014 e 2015 :

$\mathrm{O}$ número de artigos que registrou queda no número de citações entre as coletas realizadas em 2014 e 2015 foi de 53, o que representa um total de 2,9\% da amostra. Apenas duas revistas escaparam deste fenômeno: a Revista de Contabilidade da UFBA e a Revista de Contabilidade e Controladoria (UFPR). Em 31 casos a queda foi pequena, de apenas uma 


\section{GOOGLE ACADÊMICO: UMA OPÇÃO PARA ANÁLISE DE CITAÇÕES DOS PERIÓDICOS \\ BRASILEIROS DE CONTABILIDADE? \\ DOI: http://dx.doi.org/10.5007/1983-4535.2020v13n2p140}

citação. Já em 22 casos o número foi mais significante, partindo de uma queda de duas citações até um incrível número de 11 citações.

Tabela 2 Queda do número de citações registrados da coleta de 2014 para 2015

\begin{tabular}{|c|c|c|c|c|c|c|c|c|c|}
\hline Revista & -1 & -2 & -3 & -4 & -5 & -8 & -9 & -11 & Total \\
\hline BASE & 4 & 1 & 2 & & & & & & 7 \\
\hline Brazilian Business Review - BBR & 3 & 2 & & 1 & & & & & 6 \\
\hline Contabilidade Vista e Revista & 3 & 1 & 1 & 1 & & & 1 & & 7 \\
\hline Contabilidade, Gestão e Governança & 2 & & & & & & & 1 & 3 \\
\hline $\begin{array}{l}\text { Contextus - Revista Contemporânea de Economia e } \\
\text { Gestão }\end{array}$ & 2 & 1 & 1 & 2 & & & & & 6 \\
\hline Revista Brasileira de Gestão de Negócios & 3 & & 1 & & & & & & 4 \\
\hline Revista Contabilidade e Finanças & 1 & 1 & & & & & & & 2 \\
\hline Revista Contemporânea de Contabilidade & 3 & & & & & & & & 3 \\
\hline $\begin{array}{l}\text { Revista de Contabilidade do Mestrado em Ciências } \\
\text { Contábeis da UERJ }\end{array}$ & 2 & & & & & & & & 2 \\
\hline Revista de Contabilidade e Organizações & 1 & 1 & & & & & & & 2 \\
\hline Revista de Informação Contábil & 3 & 1 & & & & & & & 4 \\
\hline Revista Universo Contábil & 4 & & & & 1 & 1 & & & 6 \\
\hline Sociedade, Contabilidade e Gestão & & & 1 & & & & & & \\
\hline Total & 31 & 8 & 6 & 4 & 1 & 1 & 1 & 1 & 53 \\
\hline
\end{tabular}

Fonte: elaborada pelos autores (2017).

A Tabela 3 evidencia a mesma informação que a Tabela 2, mas para as diferenças encontradas entre os anos de 2015 e 2016 :

Tabela 3 Queda do número de citações registrados da coleta de 2015 para 2016

\begin{tabular}{l|c|c|c|c|c|c|c|c|c|c}
\multicolumn{1}{c|}{ Revista } & $\mathbf{- 1}$ & $\mathbf{- 2}$ & $\mathbf{- 3}$ & $\mathbf{- 4}$ & $\mathbf{- 5}$ & $\mathbf{- 7}$ & $\mathbf{- 1 4}$ & $\mathbf{- 2 2}$ & $\mathbf{- 2 3}$ & Total \\
\hline BASE & 2 & 1 & 1 & & & & & & 4 \\
\hline Brazilian Business Review - BBR & 0 & & & & & 1 & & & & 1 \\
\hline Contabilidade, Gestão e Governança & 4 & 1 & & & & & & & & 5 \\
\hline Revista Brasileira de Gestão de Negócios & 2 & & 1 & & 1 & & 1 & & & 5 \\
\hline Revista Contabilidade e Finanças & 2 & 1 & & & & & & 1 & 4 \\
\hline Revista Contemporânea de Contabilidade & 1 & 1 & & & & & & & & 2 \\
\hline Revista de Contabilidade da UFBA & 1 & & & 1 & & & & & & 2 \\
\hline $\begin{array}{l}\text { Revista de Contabilidade do Mestrado em } \\
\text { Ciências Contábeis da UERJ }\end{array}$ & & & & & & & & & 1 \\
\hline Revista de Contabilidade e Controladoria & & & & & & & & & & \\
\hline Revista de Contabilidade e Organizações & 1 & & & 1 & & & & & & 2 \\
\hline Revista de Informação Contábil & & & & & & & & 1 & 2 \\
\hline Revista Universo Contábil & 5 & 2 & & 1 & & & & & & 8 \\
\hline Sociedade, Contabilidade e Gestão & 4 & & & & & & & & & 4 \\
\hline Total & $\mathbf{2 4}$ & $\mathbf{6}$ & $\mathbf{3}$ & $\mathbf{3}$ & $\mathbf{1}$ & $\mathbf{1}$ & $\mathbf{1}$ & $\mathbf{1}$ & $\mathbf{1}$ & $\mathbf{4 1}$ \\
\hline
\end{tabular}

Fonte: elaborada pelos autores (2017).

O número de artigos que registrou queda no número de citações entre a coleta de 2015 e 2016 foi de 41, o que representa 2,3\% da amostra. A queda de apenas uma citação ocorreu em 24 artigos enquanto que uma queda maior de uma citação ocorreu nos demais 17 artigos 


\section{GOOGLE ACADÊMICO: UMA OPÇÃO PARA ANÁLISE DE CITAÇÕES DOS PERIÓDICOS \\ BRASILEIROS DE CONTABILIDADE? \\ DOI: http://dx.doi.org/10.5007/1983-4535.2020v13n2p140}

restantes. As maiores quedas registradas foram de 22 e 23 citações, número bastante superior a maior queda registrada entre os anos de 2014 e 2015, que foi de apenas onze. A Tabela 4, por fim, sintetiza as quedas de citações entre os anos de 2014 e 2016:

Tabela 4 Queda do número de citações registrados da coleta de 2014 para 2016

\begin{tabular}{l|c|c|c|c|c|c|c|c|c|c}
\multicolumn{1}{c|}{ Revista } & $\mathbf{- 1}$ & $\mathbf{- 2}$ & $\mathbf{- 3}$ & $\mathbf{- 5}$ & $\mathbf{- 7}$ & $\mathbf{- 8}$ & $\mathbf{- 9}$ & $\mathbf{- 1 0}$ & $\mathbf{- 1 3}$ & Total \\
\hline Brazilian Business Review - BBR & 4 & & & & & & 1 & & & 5 \\
\hline Contabilidade Vista e Revista & 2 & & 1 & & & & 1 & & & 4 \\
\hline Contabilidade, Gestão e Governança & 0 & & & & & 1 & & & & 1 \\
\hline $\begin{array}{l}\text { Contextus - Revista Contemporânea de } \\
\text { Economia G Gestão }\end{array}$ & 1 & 1 & & & & & & & & 2 \\
\hline Revista Brasileira de Gestão de Negócios & 2 & & 1 & & & & & & & 3 \\
\hline Revista Contabilidade e Finanças & 0 & & & & & & & & 1 & 1 \\
\hline Revista Contemporânea de Contabilidade & 2 & & & & & & & & & 2 \\
\hline Revista de Contabilidade e Organizações & 0 & 1 & & & & & & 1 & & 2 \\
\hline Revista de Informação Contábil & 0 & 1 & & & & & & & & 1 \\
\hline Revista Universo Contábil & 3 & & & 1 & 1 & & & & & 5 \\
\hline Sociedade, Contabilidade e Gestão & 2 & & 1 & & & & & & & 3 \\
\hline Total & $\mathbf{1 6}$ & $\mathbf{3}$ & $\mathbf{3}$ & $\mathbf{1}$ & $\mathbf{1}$ & $\mathbf{1}$ & $\mathbf{2}$ & $\mathbf{1}$ & $\mathbf{1}$ & $\mathbf{2 9}$ \\
\hline
\end{tabular}

Fonte: elaborada pelos autores (2017).

A queda do número de citações dos artigos registrada entre a coleta realizada entre 2014 e 2016 ocorreu em 29 casos, o que representa 1,6\% da amostra. Destes, 16 apresentaram uma queda de apenas uma citação e os demais 13 apresentaram quedas entre 2 e 13 citações. Entre a primeira e a terceira coleta de dados, também houve um aumento do número de revistas que apresentaram nenhum caso de queda de citações.

Em uma perspectiva mais ampla, nota-se que há uma tendência decrescente do número de artigos com queda do número de citações o que nos leva a interpretar que o algoritmo de busca de artigos e contagem das citações do Google Acadêmico se tornou mais confiável no decorrer do período analisado.

Não é possível fazer a comparação de citações dos artigos entre o WoS, o Scopus e o Google Acadêmico com artigos de periódicos brasileiros, comparação que é feita em vários artigos da literatura. Isso ocorre porque, dos 25 periódicos de Contabilidade indexados dentro da coleção principal do WoS, nenhum é brasileiro. A Tabela 5 mostra os dois periódicos brasileiros de negócios indexadas no WoS:

Tabela 5 Revistas brasileiras de Business indexadas na coleção principal do WoS

\begin{tabular}{l|c|c|c}
\hline \multicolumn{1}{c|}{ Revista } & Período & N. Artigos & Journal Impact Factor 2016 \\
\hline Custos e Agronegócio On Line & $2009-2016$ & 257 & 0,162 \\
\hline RBGN - Revista Brasileira de Gestão de Negócios & $2008-2016$ & 256 & 0,153 \\
\hline
\end{tabular}

Fonte: elaborada pelos autores (2017). 


\section{GOOGLE ACADÊMICO: UMA OPÇÃO PARA ANÁLISE DE CITAÇÕES DOS PERIÓDICOS \\ DOI: http://dx.doi.org/10.5007/1983-4535.2020v13n2p140}

A RBGN, então classificada como revista brasileira de Contabilidade na amostra desta pesquisa, aparece como revista de Negócios no WoS. No entanto, a partir de 2014, a base do Scielo Citation Index foi integrada ao WoS por meio de uma parceria da Fapesp com a Thomson Reuters (Packer, 2014). Desta forma, as revistas indexadas no Scielo também possuirão os indicadores calculados para o WoS. No entanto, apenas a Revista de Contabilidade \& Finanças e Revista Brasileira de Gestão de Negócios estão disponíveis no Scielo e, como somente os números de 2016 e 2017 da RBGN estão indexados, a mesma ainda não possui o Fator de Impacto calculado. O Fator de Impacto de dois anos, para o ano de 2016, da Revista de Contabilidade \& Finanças é de 0,1042.

A base Scopus, por sua vez, indexa um total de 73 revistas de Contabilidade, o que aumenta bastante a cobertura de artigos em relação ao WoS. No entanto, as únicas revistas brasileiras indexadas são as apresentadas na Tabela 6 .

Tabela 6 Revistas brasileiras de Contabilidade indexadas no Scopus

\begin{tabular}{l|c|c|c}
\hline \multicolumn{1}{c|}{ Revista } & Período & N. Artigos & SJR \\
\hline Custos e Agronegócio & $2009-2016$ & 398 & 0,201 \\
\hline Revista Brasileira de Gestão de Negócios & $2004-2016$ & 368 & 0,181 \\
\hline Revista Contabilidade e Finanças & $2015-2016$ & 39 & 0,111 \\
\hline
\end{tabular}

Fonte: elaborada pelos autores (2017).

No Scopus estão indexadas as três revistas listadas na Tabela 6 em que também se apresentam o SJR. Nota-se que apenas duas revistas da amostra desta pesquisa encontram-se na Scopus e que a Revista Contabilidade \& Finanças possui um número muito pequeno de artigos na base para que se permita a comparação com as citações contadas pelo Google Acadêmico.

Todavia, das quinze revistas que compõem a amostra desta pesquisa, onze tem o Fator de Impacto de 2013 calculado na Spell. Desta forma, procedeu-se a uma comparação do Fator de Impacto de 5 anos do Spell de 2013 com a média de citações dos artigos de 2008 a 2012 do Google Acadêmico, conforme mostra a Tabela 7.

Pode-se observar que o Fator de Impacto de 5 anos do Spell é menor que a média de citações dos artigos de 2008 a 2012 em todas as revistas, o que vai ao encontro da literatura que afirma que o Google Acadêmico é mais abrangente que as bases fechadas. Também podese observar que as quatro revistas com maior Fator de Impacto de 5 anos do Spell são as mesmas revistas com as maiores médias de citação do Google Acadêmico, exatamente, na mesma ordem: Revista Contabilidade \& Finanças em $1^{\circ}$, Contabilidade Vista \& Revista em 
$2^{\circ}$, Revista de Contabilidade e Organizações em $3^{\circ}$ e Revista Universo Contábil em $4^{\circ}$. A partir da Brazilian Business Review as posições não mantém mais a equidade dado que ela aparece em $5^{\mathrm{a}}$ posição no Spell e em $8^{\circ}$ no Google Acadêmico.

Tabela 7 Comparação entre o Fator de Impacto de 5 anos do Spell em 2013 com a média de citações dos artigos de 2008 a 2012 do Google Acadêmico

\begin{tabular}{|c|c|c|c|c|}
\hline Revista & Spell & \# & $\begin{array}{c}\text { Google } \\
\text { Acadêmico }\end{array}$ & \# \\
\hline BASE & 0,235 & 8 & 1,901 & 7 \\
\hline Brazilian Business Review - BBR & 0,312 & 5 & 1,853 & 8 \\
\hline Contabilidade Vista e Revista & 0,558 & 2 & 2,805 & 2 \\
\hline Contabilidade, Gestão e Governança & 0,272 & 7 & 1,933 & 6 \\
\hline Contextus - Revista Contemporânea de Economia e Gestão & 0,097 & 11 & 0,886 & 10 \\
\hline Revista Brasileira de Gestão de Negócios - RBGN & 0,306 & 6 & 1,964 & 5 \\
\hline Revista Contabilidade \& Finanças & 1,156 & 1 & 7,641 & 1 \\
\hline Revista de Contabilidade do Mestrado em Ciências Contábeis da UERJ & 0,192 & 9 & 0,765 & 11 \\
\hline Revista de Contabilidade e Organizações & 0,434 & 3 & 2,592 & 3 \\
\hline Revista Universo Contábil & 0,406 & 4 & 2,191 & 4 \\
\hline Sociedade, Contabilidade e Gestão & 0,115 & 10 & 1,067 & 9 \\
\hline
\end{tabular}

Fonte: elaborada pelos autores (2017).

Para identificar a relação destes índices procedeu-se a uma análise exploratória por meio do diagrama de dispersão.

Figura 1 Diagrama de dispersão entre as taxas de citação segundo o Spell e o Google Acadêmico Fonte: elaborada pelo autor (2017).

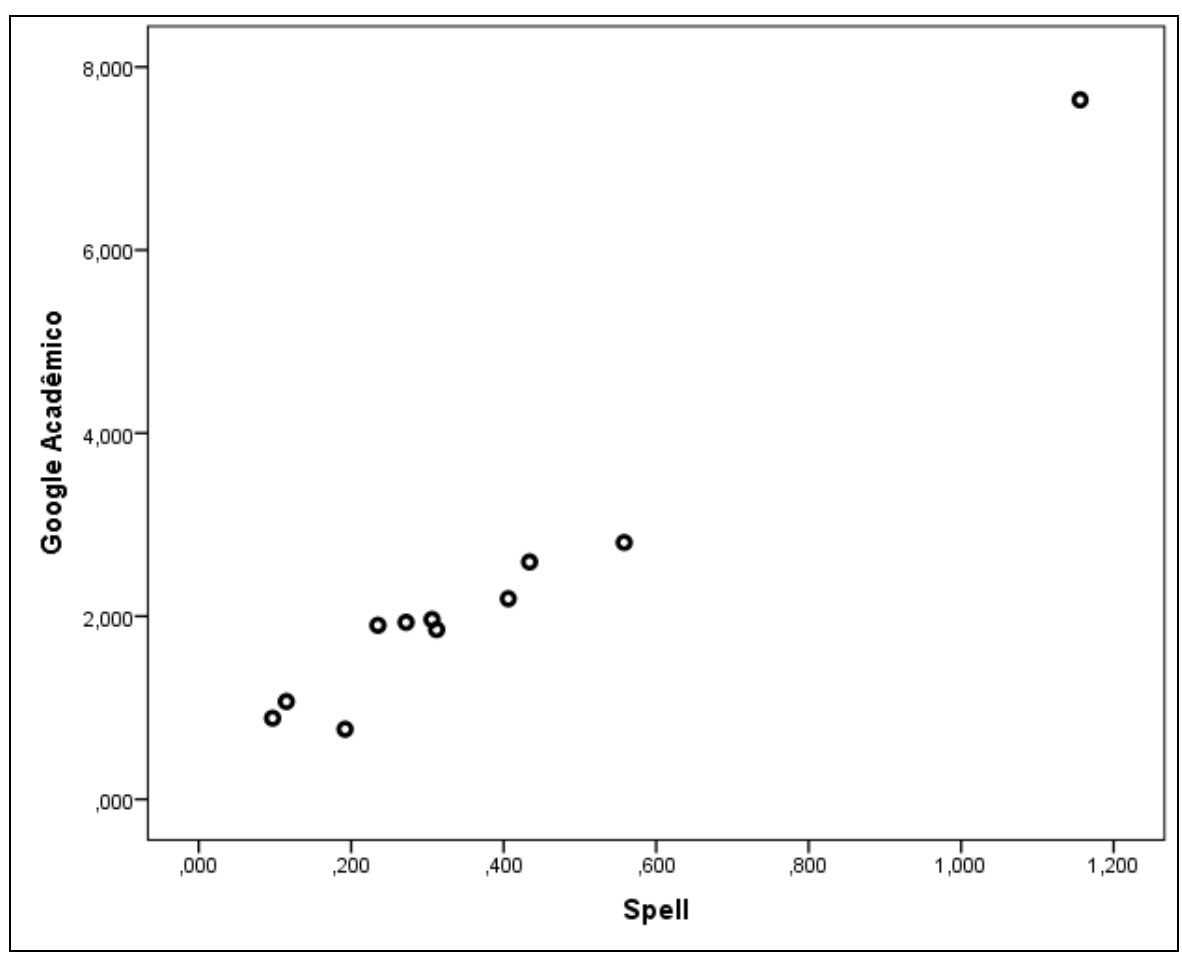

Fonte: elaborada pelos autores (2017). 


\section{GOOGLE ACADÊMICO: UMA OPÇÃO PARA ANÁLISE DE CITAÇÕES DOS PERIÓDICOS \\ BRASILEIROS DE CONTABILIDADE? \\ DOI: http://dx.doi.org/10.5007/1983-4535.2020v13n2p140}

A Figura 1 mostra que há uma associação aparentemente linear entre as duas medidas de forma que o passo seguinte foi analisar a correlação entre as duas medidas. Para tanto fezse inicialmente o teste de normalidade de Shapiro-Wilk, dado que a amostra possui $\mathrm{n}<30$ (Fávero \& Belfiori, 2017), para se proceder a escolha da medida de correlação.

Tabela 8 Testes de normalidade Shapiro-Wilk

\begin{tabular}{l|c|c|c}
\multicolumn{1}{c|}{ Variável } & Estatística & gl & Sig. \\
\hline Fator de Impacto de 5 anos do Spell & 0,781 & 11 & 0,005 \\
\hline Média de citações dos artigos entre de 2008 a 2012 do Google Acadêmico & 0,675 & 11 & 0,000 \\
\hline
\end{tabular}

Fonte: elaborada pelos autores (2017).

Os resultados dos testes rejeitam a hipótese nula de que as distribuições sejam normais, a um nível de significância de 5\%, de modo que a medida de correlação não pode ser paramétrica. Triola (2017) alerta que é um erro comum envolvendo correlação de Pearson o uso de médias pois as médias suprimem variação individual e podem aumentar o coeficiente. Diante disto, optou-se por utilizar o coeficiente de correlação $r_{s}$ - rho de Spearman, sobre o qual Field (2009) e Triola (2017) afirmam ser uma opção quando os dados são não-normais. Esta escolha foi realizada porque segundo Sampieri, Collado e Lucio (2013) o coeficiente $r_{s}$ de Spearman é mais adequado do que o tau de Kendall quando os dados são contínuos e sem um número grande de empates nos postos. A interpretação do coeficiente rho de Spearman é similar ao $r$ de Pearson (Barbetta, 2006; Sampieri, Collado \& Lucio, 2013).

Tabela 9 Matriz de correlação de Spearman

\begin{tabular}{|c|c|c|c|c|}
\hline & & & Spell & Google Acadêmico \\
\hline rho de Spearman & Spell & $\begin{array}{c}\text { Coeficiente de } \\
\text { correlação } \\
\text { Sig. (bilateral) } \\
\text { N }\end{array}$ & $\begin{array}{c}1,000 \\
11\end{array}$ & $\begin{array}{c}0,918^{* *} \\
0,000 \\
11\end{array}$ \\
\hline & Google Acadêmico & $\begin{array}{c}\text { Coeficiente de } \\
\text { correlação } \\
\text { Sig. (bilateral) } \\
\text { N }\end{array}$ & $\begin{array}{c}0,918 \\
0,000 \\
11 \\
\end{array}$ & $\begin{array}{r}1,000 \\
11 \\
\end{array}$ \\
\hline
\end{tabular}

Fonte: elaborada pelos autores (2017).

A significância do teste ( $\mathrm{p}$-valor $=0,000$ ) leva a rejeição da hipótese nula de que não há correlação entre as medidas segundo o Spell e o Google Acadêmico, a um nível de significância de 5\% (Triola, 2017). O coeficiente $r_{s}=0,918$ demonstra que as taxas de citação calculadas pelo Spell estão fortemente correlacionadas com as taxas de citação segundo o Google Acadêmico (Sampieri, Collado \& Lucio, 2013; Triola, 2017). 


\section{GOOGLE ACADÊMICO: UMA OPÇÃO PARA ANÁLISE DE CITAÇÕES DOS PERIÓDICOS \\ BRASILEIROS DE CONTABILIDADE? \\ DOI: http://dx.doi.org/10.5007/1983-4535.2020v13n2p140}

$\mathrm{Na}$ Tabela 10, faz-se uma análise da razão entre a média de citações dos artigos de 2008 a 2012 do Google Acadêmicos e o Fator de Impacto de 5 anos do Spell de 2013:

Tabela 10 Razão entre a média de citações dos artigos de 2008 a 2012 do Google Acadêmico e o Fator de Impacto de 5 anos do Spell de 2013

\begin{tabular}{l|c|c|c}
\multicolumn{1}{c|}{ Revista } & Spell & $\begin{array}{c}\text { Google } \\
\text { Acadêmico }\end{array}$ & Razão \\
\hline BASE & 0,235 & 1,901 & 8,089 \\
\hline Brazilian Business Review - BBR & 0,312 & 1,853 & 5,938 \\
\hline Contabilidade Vista e Revista & 0,558 & 2,805 & 5,027 \\
\hline Contabilidade, Gestão e Governança & 0,272 & 1,933 & 7,105 \\
\hline Contextus - Revista Contemporânea de Economia e Gestão & 0,097 & 0,886 & 9,131 \\
\hline Revista Brasileira de Gestão de Negócios - RBGN & 0,306 & 1,964 & 6,419 \\
\hline Revista Contabilidade \& Finanças & 1,156 & 7,641 & 6,61 \\
\hline Revista de Contabilidade do Mestrado em Ciências Contábeis da UERJ & 0,192 & 0,765 & 3,986 \\
\hline Revista de Contabilidade e Organizações & 0,434 & 2,592 & 5,972 \\
\hline Revista Universo Contábil & 0,406 & 2,191 & 5,397 \\
\hline Sociedade, Contabilidade e Gestão & 0,115 & 1,067 & 9,281 \\
\hline
\end{tabular}

Fonte: elaborada pelos autores (2017).

A razão calculada entre os dados do Google Acadêmico e do Spell indica que o Google Acadêmico recuperou um número de citações de 3,98 (Revista de Contabilidade do Mestrado em Ciências Contábeis da UERJ) a 9,28 vezes (Sociedade, Contabilidade e Gestão) maior que o Spell demonstrando que a abrangência do Google Acadêmico é bastante superior ao Spell. No entanto, como indicou o coeficiente de correlação de Pearson, os dois índices são bastante relacionados.

O resultado de que os motores de busca do Google Acadêmico recuperam mais citações que uma base fechada, que é o Spell, discorda da pesquisa de Jacso (2005a); Jacso (2005b); e Bakkalbasi et al. (2006); e concorda com as pesquisas de Noruzi (2005); Bar-Ilan (2008); Kousha e Thelwall (2008); Martell (2009); Onyancha (2009); Bar-Ilan (2010);

Mingers e Lipitakis (2010); Sember, Marijan e Petrak (2010); Amara e Landry (2012); Bergman (2012); e Harzing (2013), contribuindo para a construção do julgamento sobre a confiabilidade do Google Acadêmico como forma de mensurar as citações.

\section{CONCLUSÕES}

Esse artigo foi desenvolvido para identificar se o Google Acadêmico é uma opção plausível para a contagem de citações dos artigos de contabilidade brasileiros. A justificativa para o desenvolvimento desta pesquisa é o fato de que a maioria das revistas de Contabilidade brasileiras não estão indexadas no Web of Science ou no Scopus o que inviabiliza a sua 
análise nos moldes das pesquisas conduzidas em outras áreas das ciências, no exterior. Para responder a questão de pesquisa, os pesquisadores acompanharam as taxas de citação em 2014, 2015 e 2016 de 1.810 artigos publicados por quinze revistas de contabilidade entre os anos de 2007 e 2012. Os principais achados da pesquisa foram que:

- A taxa de artigos não encontrados pelo Google Acadêmico apresentou uma tendência de queda ao longo do período, caindo de 2,8\% da amostra em abril de 2014 para $0,9 \%$ da amostra em abril de 2016.

- A frequência de artigos cujo número de citações caiu, de 2014 para 2015, foi de 2,9\% da amostra. A frequência de artigos cujo número de citações caiu de 2015 para 2016, foi de $2,3 \%$ da amostra. Já a frequência de artigos cuja contagem de citações caiu entre 2014 e 2016 foi de $1,6 \%$ da amostra.

- A média das citações dos artigos publicados entre 2008 e 2012 por revista segundo o Google Acadêmico é de 3 a 9 vezes maior que o Fator de Impacto de 5 anos do Spell de 2013.

- O coeficiente de correlação de postos de Spearman entre o Fator de Impacto de 5 anos do Spell e a média de citações das citações dos artigos publicados entre 2008 e 2012 por revista é de $r=0,918$.

Estas análises suportam a conclusão de que o Google Acadêmico se apresenta como uma opção relativamente segura, embora não perfeita, para a análise de citações das pesquisas contábeis brasileiras. Esta conclusão é importante porque permite que análises de citações sejam desenvolvidas sobre artigos brasileiros de Contabilidade.

Embora as médias de citações calculadas com dados do Google Acadêmico sejam muitas vezes maior que o Fator de Impacto do Spell, a alta correlação entre os dois mostram que os dois guardam uma relação altamente positiva, e que feitas as devidas ressalvas, o Google Acadêmico pode ser usado para calcular citações das mais de vinte outras revistas de Contabilidade brasileiras não indexadas pelo Spell.

Como sugestão para pesquisas futuras, sugere-se medir a confiabilidade da contagem de citações do Google Acadêmico por meio da averiguação de cada uma das citações encontradas pelo buscador para identificar se não se trata da atribuição equivocada de citação ou a ocorrência de duplicidades na contagem. Sugere-se também classificar a origem das citações para identificar a proporção de citações derivadas de teses, dissertações, monografias ou anais de eventos que as bases como os Spell não captam. Para as revistas brasileiras agora incluídas nos indexadores internacionais, pode-se fazer uma comparação das citações e dos 
fatores de impacto de revistas, artigos e autores, devendo, para isso, aguardar-se alguns anos para que se estabeleçam os fatores de citações para 3 e 5 anos.

\section{REFERÊNCIAS}

AMARA, N.; LANDRY, R. Counting citations in the field of business and management: Why use Google Scholar rather than the Web of Science. Scientometrics, v. 93, n. 3, p. 553-581, 2012.

ARAGÃO, I. R. B. N.; OLIVEIRA, J. R. S.; LIMA, G. A. S. F. Ressonância de Artigos e Fator de Impacto de Periódicos Brasileiros de Contabilidade. Revista de Educação e Pesquisa em Contabilidade (REPeC), v. 8, n. 1, p. 5-21, 2014.

BAKKALBASI, N. et al. Three options for citation tracking: Google Scholar, Scopus and Web of Science. Biomedical digital libraries, v. 3, n. 1, p. 7, 2006.

BAR-ILAN, J. Which h-index? - A comparison of WoS, Scopus and Google Scholar. Scientometrics, v. 74, n. 2, p. 257-271, 2008.

BAR-ILAN, J. Citations to the "Introduction to informetrics" indexed by WOS, Scopus and Google Scholar. Scientometrics, v. 82, n. 3, p. 495-506, 2010.

BARBETTA, P. A. Estatística aplicada às Ciências Sociais. 6. ed. Florianópolis: Ed. da UFSC, 2006.

BERGMAN, E. M. L. Finding citations to social work literature: The relative benefits of using Web of Science, Scopus, or Google Scholar. The journal of academic librarianship, v. 38, n. 6 , p. $370-379,2012$.

BORNMANN, L. et al. Convergent validity of bibliometric Google Scholar data in the field of chemistry - Citation counts for papers that were accepted by Angewandte Chemie International Edition or rejected but published elsewhere, using Google Scholar, Science Citation Index, Scopus, and Chemical Abstracts. Journal of informetrics, v. 3, n. 1, p. 27-35, 2009.

CHAN, K. C.; CHANG, C.; CHANG, Y. Ranking of finance journals: Some Google Scholar citation perspectives. Journal of Empirical Finance, v. 21, p. 241-250, 2013.

DELGADO-LÓPEZ-CÓZAR, E.; CABEZAS-CLAVIJO, A. Ranking journals: could Google scholar metrics be an alternative to journal citation reports and Scimago journal rank?

Learned publishing, v. 26, n. 2, p. 101-113, 2013.

FÁVERO, L. P.; BELFIORI, P. Manual de análise de dados - estatística e modelagem multivariada com Excel, SPSS e Stata. Rio de Janeiro: Elsevier, 2017.

FIELD, A. Descobrindo a estatística usando SPSS. 2. ed. Porto Alegre: Artmed, 2009. 
GARCÍA-PÉREZ, M. A. Accuracy and completeness of publication and citation records in the Web of Science, PsycINFO, and Google Scholar: A case study for the computation of $h$ indices in Psychology. Journal of the Association for Information Science and Technology, v. 61, n. 10, p. 2070-2085, 2010.

HARZING, A. A preliminary test of Google Scholar as a source for citation data: a longitudinal study of Nobel prize winners. Scientometrics, v. 94, n. 3, p. 1057-1075, 2013.

JACSO, P. As we may search - comparison of major features of the Web of Science, Scopus, and Google Scholar citation-based and citation-enhanced databases. Current Science, v. 89, n. 9, p. 1537-1547, 2005a.

JACSO, P. Comparison and analysis of the citedness scores in Web of Science and Google Scholar. Lecture Notes in Computer Science, v. 3815, p. 360-369, 2005 b.

KOUSHA, K.; THELWALL, M. Google Scholar citations and Google Web/URL citations: A multi-discipline exploratory analysis. Journal of the Association for Information Science and Technology, v. 58, n. 7, p. 1055-1065, 2007.

KOUSHA, K.; THELWALL, M. Sources of Google Scholar citations outside the Science Citation Index: A comparison between four science disciplines. Scientometrics, v. 74, n. 2, p. 273-294, 2008.

KULKARNI, A. V. et al. Comparisons of citations in Web of Science, Scopus, and Google Scholar for articles published in general medical journals. Jama, v. 302, n. 10, p. 1092-1096, 2009.

MARTELL, C. A citation analysis of college \& research libraries comparing Yahoo, Google, Google Scholar, and ISI Web of Knowledge with implications for promotion and tenure.

College \& Research Libraries, v. 70, n. 5, p. 460-473, 2009.

MEHO, L. I.; YANG, K. Impact of data sources on citation counts and rankings of LIS faculty: Web of Science versus Scopus and Google Scholar. Journal of the Association for Information Science and Technology, v. 58, n. 13, p. 2105-2125, 2007.

MINGERS, J.; LIPITAKIS, E. A. Counting the citations: a comparison of Web of Science and Google Scholar in the field of business and management. Scientometrics, v. 85, n. 2, p. 613-625, 2010.

NORUZI, A. Google Scholar: the new generation of citation indexes. Libri, v. 55, n. 4, p. 170-18, 2005.

ONYANCHA, O. B. A citation analysis of sub-Saharan African library and information science journals using Google Scholar. African Journal of Library, Archives and Information Science, v. 19, n. 2, p. 101-116, 2009. 
ORTEGA, J. L.; AGUILLO, I. F. Microsoft academic search and google scholar citations: Comparative analysis of author profiles. Journal of the Association for Information Science and Technology, v. 65, n. 6, p. 1149-1156, 2014.

PACKER, A. (2014). SciELO Citation Index no Web of Science. Disponível em http://blog.scielo.org/blog/2014/02/28/scielo-citation-index-no-web-of-science/. Acesso em: em 20 ago. 2017.

PAULY, D.; STERGIOU, K. I. Equivalence of results from two citation analyses: Thomson ISI's Citation Index and Google's Scholar service. Ethics in Science and Environmental Politics, v. 2005, p. 33-35, 2005.

PERKEL, Jeffrey. The Future of Citation Analysis. 2005. Disponível em: https://www.thescientist.com/tribute/the-future-of-citation-analysis-48241. Acesso em: 23 out. 2005.

SAMPIERI, R. H.; COLLADO, C. F.; LUCIO, M. P. B. Metodologia de pesquisa. 5. ed. Porto Alegre: Penso, 2013.

SANNI, S. A.; ZAINAB, A. N. Google Scholar as a source for citation and impact analysis for a non-ISI indexed medical journal. Malaysian Journal of Library \& Information Science, v. 15, n. 3, p. 35-51, 2010.

SCHROEDER, R. Pointing users toward citation searching: using Google Scholar and Web of Science. Libraries and the Academy, v. 7, n. 2, p. 243-248, 2007.

ŠEMBER, M.; Utrobičić, A.; Petrak, J. Croatian medical journal citation score in web of science, scopus, and google scholar. Croatian medical journal, v. 51, n. 2, p. 99-103, 2010.

SOARES, S. V.; CASA NOVA, S. P. C. O qualis reflete o impacto dos artigos de revistas brasileiras de contabilidade? Revista de Gestão, Finanças e Contabilidade, v. 6, n. 3, p. 6$23,2016$.

SOARES, S. V. Diga-me quantos te citam, e eu te direi quem és - Estudo sobre as citações no âmbito da pesquisa contábil brasileira. Tese de doutorado em Controladoria e

Contabilidade, Programa de Pós-Graduação em Controladoria e Contabilidade, Faculdade de Economia, Administração e Contabilidade, Universidade de São Paulo, São Paulo, SP, 2017.

SOUZA, J. V.; ENSSLIN, S. R.; ENSSLIN, L. Panorama dos periódicos vinculados à área contábil quanto ao reconhecimento da sociedade científica. In: CONGRESSO USP DE CONTROLADORIA E CONTABILIDADE, 12., 2012, São Paulo. Anais... São Paulo: FEA/USP, 2012.

SPELL. Impacto. Disponível em: http://www.spell.org.br/impacto/ajuda. Acesso em: 20 ago. 2017.

TRIOLA, M. F. Introdução a estatística. 12. ed. Rio de Janeiro: LTC, 2017. 